\title{
Maintaining Optimal Communication Chains in Robotic Sensor Networks using Mobility Control
}

\author{
Cory Dixon \\ Research \& Engineering Center \\ for Unmanned Vehicles \\ University of Colorado \\ Boulder, Colorado 80309-0429 \\ Email: Cory.dixon@colorado.edu
}

\author{
Eric W. Frew \\ Research \& Engineering Center \\ for Unmanned Vehicles \\ University of Colorado \\ Boulder, Colorado 80309-0429 \\ Email: Eric.frew@colorado.edu
}

\begin{abstract}
Electronic chaining is the formation, and maintenance, of a linked communication chain that maximizes the endto-end throughput using a cooperative team of mobile robotic relays. For this paper, an optimal communication chain is defined using the signal-to-noise ratio (SNR) of the communication links along the chain. By using the SNR of the individual communication channels, instead of relative position, an optimal communication chain of robotic relays is formed that is able to respond to changes in, and unexpected features of, the RF environment that is simply not possible with position based chaining solutions. Since the operating environment is generally not known a priori to deployment of a robotic sensor network, an adaptive model-free extremum seeking (ES) algorithm is presented to control the motion of $2 \mathrm{D}$ nonholonomic vehicles acting as communication relays. Even without specific knowledge of the SNR field, the ES algorithm is able to drive the team of vehicles to optimal locations with only local measures of the SNR. A specific application using unmanned aircraft is simulated to highlight the fact that the performance of the ES chaining algorithm is limited due to the performance constraints and capabilities of the individual vehicles within the chain.
\end{abstract}

\section{INTRODUCTION}

Cooperative electronic chaining is the formation of a linked communication chain using a team of robotic vehicles acting as communication relays in an ad hoc network while allowing the end nodes of the chain to move independently in an unknown, dynamic environment [1]. Electronic chaining utilizes the fact that with networked robotic vehicles the quality of a wireless communication chain is directly influenced by the motion and location of the vehicles within the radio propagation environment. Thus, controlling the location of the vehicles based on a measure of communication performance can used to directly improve the communication performance along the network chain. Inherent in the concept of electronic chaining is the repair of disconnected networks using robotic relays. The difference is that in electronic chaining the goal is more than providing connectivity, it is to provide optimal connectivity of the networks through the relay.

This paper presents a definition of an optimal communication chain using the signal-to-noise ratio (SNR) of the radio frequency (RF) communication links between the robotic nodes (or networks), as opposed to relative position as previously done in [2]. By moving the robotic relays based on the local gradients of the SNR fields of the relay's communication links, the communication capability of the chain can be improved regardless of the communication environment, which could include RF jamming nodes. The use of the SNR as input into a control system for robotic vehicles to improve and maintain communication performance was originally presented by the authors in [3] and was further developed using extremum seeking (ES) methods in [4].

While there has been significant work in robotic team control requiring network communications (e.g. [5]-[7]), only a small body of work (see [2], [8]-[10]) exists that explicitly incorporates communication objectives into larger multiobjective control framework. Although the goal is to optimize network parameters in these works, the performance metrics are transformed into position based constraints and cost functions.

For example, in [2] the authors make the claim that the set of optimal positions of the relay nodes lies entirely on the line between the source and destination nodes, and that the relay nodes must be evenly spaced along this line. However, as will be shown in this paper, in a physical environment the assumptions required for position based control are typically invalid since localized noise sources, terrain affects, power differences, and antenna patterns will cause the optimal location to move off of the center line between the two end nodes and possibly away from the geometric center point of the line. By defining the optimal communication chain in a more generalized sense using the SNR, the communication chain that is formed is more robust and can respond to changes in the RF environment.

If the structure of the SNR field is known, it will be shown in this paper that a decentralized controller, based on the generalized gradient of a local performance function, will drive the robotic nodes to optimal relay locations. If however the structure of the SNR field and the local environment is not known, as is typically the case in real-world deployments of robotic networks, then an adaptive model-free controller that estimates the gradient of the SNR fields is required. To this end, this paper also presents a decentralized ES controller that has been designed for use on planar nonholonomic vehicles for electronic chaining. 
Fundamental to the ES chaining algorithm is that to estimate the gradient of the communication performance field, cyclic motion of the vehicle is required. For generality in application to different vehicle types, a bicycle-like kinematic vehicle model [11], exhibiting Dubins' vehicle constraints [12], is assumed and a Lyapunov Guidance Vector Field (LGVF) controller [13] is used to provide the cyclic motion by driving the vehicle to a globally stable limit cycle (i.e. a circular orbit) about a virtual center point.

While ES algorithms have been presented in [14] to drive a nonholonomic vehicle in a sampled environment, driving the vehicle directly using the ES framework limits the application to limited vehicle types. Specifically the ES algorithm in [14] can only be used on vehicles that can go forward and backwards as the controller modulates the forward velocity of the vehicle, while holding a constant turn rate. The use of the LGVF controller to generate a circular motion, with forward vehicle velocity and bounded turn rate capabilities, can be applied to a much wider class of robotic vehicles; from simple point mass models to unmanned aircraft. In addition, the circular motion of the vehicle due to the LGVF is more natural for certain vehicles such as unmanned aircraft.

The authors of [14] have proposed a second part to their work (but is still unpublished) where the velocity of the vehicle is held constant and the turning rate of the vehicle is modulated by the ES algorithm. This resulting motion of this controller is a forward motion of the vehicle with a "wiggle." The idea being that if one can mount a sensor on a long boom to the vehicle, a small wiggle of the vehicle will result in a large displacement of the sensor in the sampled environment. However, on some vehicles it is not practical to mount a long boom sticking out the nose of the vehicle. In addition, there are some environments where the displacement of the sensor on a boom will still not provide a large enough displacement for the sensor to measure the change in the sampled environment, either due to the field structure or the sensor resolution. Thus, driving the vehicle in a circular motion, about a virtual center point, provides a more generic ES framework for mobility control of vehicles.

\section{Electronic Chaining Problem Statement}

For a linked network chain, independent of the communication protocols used, the achievable chain capacity can be directly related to the individual link capacities along the chain. The relation of the local link capacity to the full chain capacity is defined by the type of network being considered. In this paper a Cascaded relay network [15] is considered. A cascaded relay network represents a network model where the relays can transmit and receive at the same time (and in the same bandwidth). This is possible if the relay has two antennas: one for receiving and one transmitting. While this is typically not the case for cheap wireless network nodes that use a single omnidirectional antenna, this network model lends itself to decentralized control quite well, as will be shown.

Because of the assumption of simultaneous transmission and reception of signals by a relay, the throughput capacity of a cascaded network chain, $T$, is limited by the link with smallest throughput capacity. Figure 1 provides a graphical example of the problem where the link between nodes 3 and 4 is limited to 1 megabit per second ( $\mathrm{Mbps}$ ), either due to distance or environmental noise, and the rest of the chain has a $2 \mathrm{Mbps}$ link capacity.

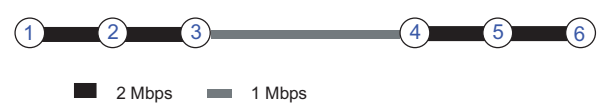

Fig. 1. The overall chain capacity of a cascaded network is directly limited to the link with the smallest capacity. For this chain, the throughput from node 1 to node 6 is only 1 Mbps.

It is clear from the figure that even if node 1 tries to transmit at $2 \mathrm{Mbps}$ to node 6 , that the link between nodes 3 and 4 will limit the resulting throughput to node 6 from node 1 to be $1 \mathrm{Mbps}$. Thus in a communication chain with mobile nodes, the nodes should move so as to find the maximum chain throughput capacity,

$$
T^{*}=\max T=\max \min T_{i j}
$$

where $T^{*}$ is the globally optimal communication throughput capacity for a chain in an unknown environment with localized noise sources (e.g. jamming sources).

It should be stated that the goal of this work is to improve and maintain communication capacity of a wireless chain. The actual throughput (or goodput) of a network chain is dependent upon the network protocols, data encoding used by the radio, signal strengths, interfering neighbors and the amount of data being sent from the source. If the source is not sending any data to the destination, then the throughput of the chain is zero, and thus the gradient of the throughput is also zero. However the communication chain should still respond to the movements of the end nodes, to maintain an optimal networked chain for when the source node does send data. Thus, for the purpose of this paper, throughput is shorthand for the throughput capacity of the chain, and is not the actual data sent through the chain. In addition, it is assumed that a fixed number of relays is to be used in the chain and that this number is determined by a higher level controller, which determines the optimal number of relay nodes that should be used.

\section{A. Radio Frequency $(R F)$ Environment}

The signal-to-noise ratio (SNR) of an RF communication link is defined as

$$
S_{i j}\left(\mathbf{p}_{i}, \mathbf{p}_{j}\right)=\frac{P_{i j}\left(\mathbf{p}_{i}, \mathbf{p}_{j}\right)}{N\left(\mathbf{p}_{i}\right)}
$$

where $P_{i j}\left(\mathbf{p}_{i}, \mathbf{p}_{j}\right)$ is the power received by node $i$ at position $\mathbf{p}_{i} \in \mathbb{R}^{2}$ from the transmission of node $j$, located at $\mathbf{p}_{j} \in \mathbb{R}^{2}$. $N\left(\mathbf{p}_{i}\right)$ is the environmental noise seen by node $i$ at location $\mathbf{p}_{i}$, and includes thermal and interference noise. For simplification of notation let $S_{i j}=S_{i j}\left(\mathbf{p}_{i}, \mathbf{p}_{j}\right)$.

The Shannon-Hartley Theorem states that the channel capacity $C$, which is the theoretical maximum rate of clean (or 
arbitrarily low bit error rate) data that can be sent with a given average SNR is [16]

$$
C_{i j}\left(\mathbf{p}_{i}, \mathbf{p}_{j}\right)=B \log _{2}\left(1+S_{i j}\left(\mathbf{p}_{i}, \mathbf{p}_{j}\right)\right),
$$

where $B$ is the bandwidth of the channel, and $C_{i j}\left(\mathbf{p}_{i}, \mathbf{p}_{j}\right)$ is the channel capacity for node $j$ at position $\mathbf{p}_{j}$ transmitting to node $i$ at position $\mathbf{p}_{i}$. The Shannon-Hartley Theorem provides a very useful relation in that the maximum achievable rate is related to the SNR of the channel. By increasing the SNR of a wireless channel, the ability of the channel to send more data is increased, providing a higher capacity capability.

For this paper it is assumed that the RF environment can contain localized noise sources such that $N\left(\mathbf{p}_{i}\right) \neq N\left(\mathbf{p}_{j}\right)$, giving $S_{i j} \neq S_{j i} \Rightarrow C_{i j} \neq C_{j i}$. That is to say, even though node $i$ can receive a transmission from node $j$, node $j$ may not be able to decode a transmission from node $i$. This is a fundamental assumption for any geographic, range-based controller, and for the definition of an optimal chain given in [2].

\section{B. Optimal Communication Chain}

Let a cascaded network chain with nodes 1 through $n$, ordered by their position in the chain, be designated $N$. Let $R \subset N$ be the set of relay nodes in the network $N$, i.e. the set of relay nodes does not contain the two end nodes of the chain which move independent of the chain. The two end nodes represent the users of the communication chain and it is the goal of the mobile relay nodes to position themselves so as to obtain, and maintain, an optimal communication chain in response to the movements of the two end nodes. Thus, nodes 1 and $n$ are allowed to move freely and independently while nodes 2 through $n-1$ are mobile relays that are controlled by the electronic chaining algorithm.

Optimal bi-directional chain throughput capacity is found by maximizing the minimum individual link capacities by moving the relay nodes in the environment so that

$$
T^{*}=\max _{\mathbf{p}_{i} \in \mathbb{R}^{2}} \min _{\substack{i \in R, j \in N \\|j+i|=1}}\left\{C_{i j}\left(\mathbf{p}_{i}, \mathbf{p}_{j}\right), C_{j i}\left(\mathbf{p}_{j}, \mathbf{p}_{i}\right)\right\}
$$

Since link capacity is a monotonically increasing function of the SNR, it is desirable to introduce a measure of an optimal chain similar to Eq. 4 but based solely on the individual link SNRs as

$$
S^{*}=\max _{\mathbf{p}_{i} \in \mathbb{R}^{2}} \min _{\substack{i \in R, j \in N \\|j+i|=1}}\left\{S_{i j}\left(\mathbf{p}_{i}, \mathbf{p}_{j}\right), S_{j i}\left(\mathbf{p}_{j}, \mathbf{p}_{i}\right)\right\}
$$

Finally, the goal of electronic chaining as presented in this paper is to find

$$
\mathbf{p}_{i}^{*}=\arg \max _{\mathbf{p}_{i} \in \mathbb{R}^{2}} \min _{\substack{i \in R, j \in N \\|j+i|=1}}\left\{S_{i j}, S_{j i}\right\}
$$

in real time, using a globally stable decentralized controller based on the SNR of only neighbor links and without any specific model or knowledge of the SNR field.

\section{Decentralized Gradient Based Controller}

If the gradients of the SNR field are known, then a decentralized (localized) controller based on the generalized gradient [17] of a nonsmooth Lyapunov function can be used to drive the robotic relays to a set of optimal locations as defined by Eq. 6, with asymptotic stability [18].

Let $\mathbf{x}_{i} \in \mathbb{R}^{2}$ be the state vector for node $i$ subject to

$$
\dot{\mathbf{x}}_{i}=\mathbf{u}_{i}
$$

where $\mathbf{u}_{i}: \mathbb{R}^{2} \times \mathbb{R}^{2} \rightarrow \mathbb{R}^{2}$ is the control input to node $i$. First order dynamics are presented here for ease of discussion. Extension to higher order dynamics is possible with a correct choice of the Lyapunov function.

Define the Lyapunov candidate function $J_{i}: \mathbb{R}^{2} \times \mathbb{R}^{2} \rightarrow \mathbb{R}^{+}$ to be

$$
J_{i}\left(\mathbf{x}_{i}\right)=\max _{\substack{j \in R \\|j+i|=1}}\left\{1 / S_{i j}, 1 / S_{j i}\right\}-1 / S_{i}^{*}
$$

for the locally optimal SNR of relay $i, S_{i}^{*}$, given some location of node $i$ 's neighbors in the environment. Thus, $J_{i}\left(\mathbf{x}_{i}\right)>0$ unless $\mathbf{x}_{i}=\mathbf{p}_{i}^{*}$ for which $J_{i}\left(\mathbf{p}_{i}^{*}\right)=0$.

The performance function is presented in this form as opposed to that suggested by Eq. 5 due to the structure of the SNR fields having exponential decay with distance. Because of this, the function $f=1 / S_{i j}$ ( $S_{i j}$ being non-zero by definition) is continuous and convex about a bounded interval $B \in \mathbb{R}^{2}$ containing a critical point of $J_{i}$. From convex functional calculus, if $a(x)$ and $b(x)$ are convex functions, then so is $h(x)=\max \{a(x), b(x)\}$.

Due to the fact that localized noise sources may be present in the environment, it is possible for $J_{i}\left(\mathbf{x}_{i}\right)$ to have multiple critical points, i.e. local and global extremum points. That is to say, $\nabla J_{i}\left(\mathbf{p}_{i}^{\dagger}\right)=0$ even though $\mathbf{p}_{i}^{\dagger} \neq \mathbf{p}_{i}^{*}$. Thus, a controller based on the generalized gradient of $J_{i}$ cannot guarantee convergence to the global optimum of $J_{i}$. Only that given some initial $\mathbf{p}_{i}(0)$ in a neighborhood $\Omega$ about a critical point $\mathbf{p}_{i}^{\dagger}$, that the system will asymptotically converge to the critical point $\mathbf{p}_{i}^{\dagger}$ (note $\mathbf{p}_{i}^{*} \subseteq \mathbf{p}_{i}^{\dagger}$ ).

This is not considered to be a downfall of the gradient controller as it still drives the position of the relay to a more optimal location than can be obtained by simply placing the relay in the geographic center. If the environment does not contain localized effects, and $S_{i j}=S_{j i}$ then $\mathbf{x}_{i}^{\dagger}=$ $\mathbf{x}_{i}^{*} \Rightarrow \mathbf{X}^{\dagger}=\mathbf{X}^{*}$ and the gradient based controller is globally asymptotically stable to the global maximum $T^{*}$ at $\mathbf{X}^{*}$ since $\dot{V}(\mathbf{X})>0$ unless $\dot{V}\left(\mathbf{X}^{*}\right)=0$.

Define the decentralized feedback controller to be

$$
\mathbf{u}_{i}=-k \nabla J_{i}\left(\mathbf{x}_{i}\right)
$$

where $\nabla J_{i}\left(\mathbf{x}_{i}\right)$ is the gradient of $J_{i}$, and $k \in \mathbb{R}^{+}$is the controller gain. It should be noted that since Eq. 8 is nonsmooth, the above equation abuses notation as the gradient here is really the generalized gradient of $J_{i}$. The stability and stabilization of dynamic systems using feedback of the form of Eq. 9 for nonsmooth Lyapunov functions has been well studied and presented in [18]. 
From [17], let $f: \mathbb{R}^{m} \rightarrow \mathbb{R}$ be locally lipschitz. Then the generalized gradient of $f$ is defined as

$$
\partial f(\mathbf{x})=\operatorname{co}\left\{\lim _{\substack{h_{i} \rightarrow 0 \\ i \rightarrow \infty}} \nabla f\left(\mathbf{x}+\mathbf{h}_{i}\right)\right\}
$$

where $\operatorname{co}\{\}$ denotes the convex closure of the set. If $f$ is differentiable at $\mathbf{x} \in \mathbb{R}^{m}$, then $\partial f(\mathbf{x})=\{\nabla f(\mathbf{x})\}$. Let $\Omega_{f}$ denote the set of points where $f$ fails to be differentiable. Then for the purpose of this paper if $0 \in \partial J_{i}(\mathbf{x})$ at $\mathbf{x} \in \Omega_{f}$, then $\nabla J_{i}(\mathbf{x})$ will be taken to be zero. If however the zero vector is not within the set, then $\nabla J_{i}(\mathbf{x})$ can be chosen to be the any vector $v \in \partial J_{i}$. To be consistent, the least-norm element of $\partial J_{i}$ is chosen to represent the gradient of the system on $\Omega_{f}$. The notation of Eq. 8 will be used in this paper for readability as it is intuitive for the reader.

It is desirable to show the differences between the decentralized controller and a centralized one, working on a globally valued performance function, to show that that the decentralized controller solves the global optimization problem, and does so more efficiently. Take the Lyapunov candidate function for the localized controller to be $V_{i}=J_{i}\left(\mathbf{p}_{i}\right)$ from Eq. 8. Then using the chain rule, realizing that in reality we are taking a set-valued derivative of $V_{i}$ with respect to the system of Eq. 7 ,

$$
\begin{aligned}
\dot{V}_{i} & =\partial V_{i} \cdot \frac{d \mathbf{x}_{i}}{d \mathrm{t}}=\nabla V_{i} \cdot \dot{\mathbf{x}}_{i} \\
& =\nabla V_{i} \cdot\left(-k \nabla J_{i}\right) \\
& =-k\left\|\nabla J_{i}\right\|^{2} .
\end{aligned}
$$

Since $V_{i}\left(\mathbf{x}_{i}\right)>0$, except at $\mathbf{x}_{i}=\mathbf{x}_{i}^{*} \Rightarrow V_{i}\left(\mathbf{x}_{i}^{*}\right)=0$, and that $\dot{V}_{i} \leq 0$, the local controller is asymptotically stable in the sense of Lyapunov (i.s.L.) for some given neighbor positions, $\mathbf{p}_{i \pm 1}$, in the operating environment.

It will now be shown that this local controller solves the global optimization problem of Eq. 6. Let $\mathbf{X}=$ $\left[\mathbf{x}_{1}, \mathbf{x}_{2}, \cdots, \mathbf{x}_{n}\right]^{T}$, and $\mathbf{U}=\left[\mathbf{u}_{1}, \mathbf{u}_{2}, \cdots, \mathbf{u}_{n}\right]^{T}$ such that

$$
\dot{\mathrm{X}}=\mathbf{U} \text {. }
$$

Define the globally valued Lyapunov candidate function to be

$$
V=J(\mathbf{X})=\max _{\substack{i \in R, j \in N \\|j+i|=1}}\left\{1 / S_{i j}, 1 / S_{j i}\right\}-1 / S^{*}
$$

for the global maximum $S^{*}$. Even though $V=$ $V\left(S_{k l}\left(\mathbf{p}_{k}, \mathbf{p}_{l}\right)\right)$, given $k, l \in N$ and $|k+l|=1$, is a function of all neighbor links in both directions, the gradient of $V$ at any one specific moment is only a function of one neighbor set, e.g. the two neighbor nodes of the link with the smallest SNR.

Let $k, l$ be the indicies of the argument of the output link from the max function in Eq. 13, e.g.

$$
S_{k l}=\min _{\substack{i \in R, j \in N \\|j+i|=1}}\left\{S_{i j}, S_{j i}\right\}
$$

such that

$$
V=1 / S_{k l}-1 / S^{*}
$$

Now taking the gradient of $V$ with respect to (w.r.t.) all node positions,

$$
\begin{aligned}
\nabla V & =\frac{\partial}{\partial \mathbf{X}} V=\frac{\partial}{\partial \mathbf{X}} \frac{1}{S_{k l}} \\
& =\left[0, \cdots, 0, \frac{\partial}{\partial \mathbf{x}_{k}} \frac{1}{S_{k l}}, \frac{\partial}{\partial \mathbf{x}_{l}} \frac{1}{S_{k l}}, 0, \cdots, 0\right]^{T} \\
& =\left[0, \cdots, 0, \nabla_{k} V_{k}, \nabla_{l} V_{l}, 0, \cdots, 0\right]^{T}
\end{aligned}
$$

it is seen that at any one moment the global gradient is a function of two localized gradients. Finally, it is shown that the local controller acts to solve the global optimization problem using a Lyapunov stability argument. Applying the chain rule to $V$, and using the dynamics of Eq. 12,

$$
\begin{aligned}
\dot{V} & =\nabla V \cdot \dot{\mathbf{X}} \\
& =\nabla V \cdot \mathbf{U} \\
& =\left[0, \cdots, 0, \nabla_{k} V_{k}, \nabla_{l} V_{l}, 0, \cdots, 0\right]^{T} \cdot\left[\mathbf{u}_{1}, \mathbf{u}_{2}, \cdots, \mathbf{u}_{n}\right]^{T} \\
& =-k\left(\left\|\nabla_{k} V_{k}\right\|^{2}+\left\|\nabla_{l} V_{l}\right\|^{2}\right)
\end{aligned}
$$

Then, since $\dot{V} \geq 0$ and that $V \leq 0$ the system is asymptotically stable i.s.L. to a set of critical points of the global performance function.

Because of this, a centralized controller would only move two nodes at any given moment (or time step), while in the case of the decentralized controller every node is always responding to both of its neighbor nodes at a every time step. Thus, the decentralized version will converge to optimal relay locations faster than if the controller was ran at a central location using the global objective function.

\section{Electronic Chaining ES Controller}

It was shown in the previous section that if the local gradients are known by the nodes, then a decentralized controller (based on the localized gradients) can be used to drive individual nodes to their globally optimal locations. However, in a physical environment with unknown localized noise sources, either due to faulty nodes or jamming, the structure of the SNR field is unknown and unpredictable and therefor the gradient can not be directly deteremined. In addition, the operating environment of the nodes will have an impact on the communication performance. Which is difficult to predict prior to deployment of the system to an unknown location. Thus, a way to estimate the gradient of the performance objective in real-time, and by each mobile node, is required so that the system may be driven to optimal operating positions (setpoints).

Extremum seeking (ES) [19] controllers are adaptive, model-free controllers designed to drive the set point of a dynamic system to an optimal, but unpredictable location defined by a performance function that is only known to have an extremum point. That is, given a sufficiently smooth cost function $J: \mathbb{R} \times \mathbb{R}^{m} \rightarrow \mathbb{R}$, ES controllers seek to solve in real time the optimization problem

$$
\theta^{*}(t)=\arg \max _{\theta \in \mathbb{R}^{m}} J(t, \theta)
$$


where $J$ is an unknown, possibly time varying, cost function of the input parameter $\theta$ such that $D_{\theta} J\left(t, \theta^{*}\right)=0$ and $D_{\theta}^{2} J\left(t, \theta^{*}\right)<0^{1}$.

The standard ES algorithm works by generating a measure of the local gradient of the mapping $J(\theta)$ by injecting a perturbation signal, $\alpha \cos (\omega t)$, directly into the plant. The output of the plant will also be sinusoidal, with a DC (or constant) offset that the HPF removes. This signal is then demodulated by $\beta \sin (\omega t-\gamma)$ and low-pass filtered to obtain the gradient estimate. The gradient estimate is then used to update the estimate of the optimal location, $\hat{\theta}$. See [19], [20] for formal discussions, including stability proofs and design guidelines, on single and multivariable ES.

In two dimensions, the input into the performance function has the appearance of a circular perturbation about a moving (i.e. time varying) orbit center point. It is this specific structure that the ES algorithm presented in this paper takes advantage of in that some vehicles, like unmanned aircraft (UA), also exhibit a cyclic (circular) motion about an orbit center point when they are station keeping since they must always maintain a forward speed.

A block diagram of the decentralized ES chaining algorithm is shown in Fig. 2 and consists of a Lyapunov Guidance Vector Field Controller steering a 2D kinematic vehicle operating within an ad hoc network. The basic ES framework within the controller is used to estimate the gradient of the communication performance field that is used to drive the motion of the orbit center point for the LGVF controller using virtual point mass dynamics with a bounded center point velocity.

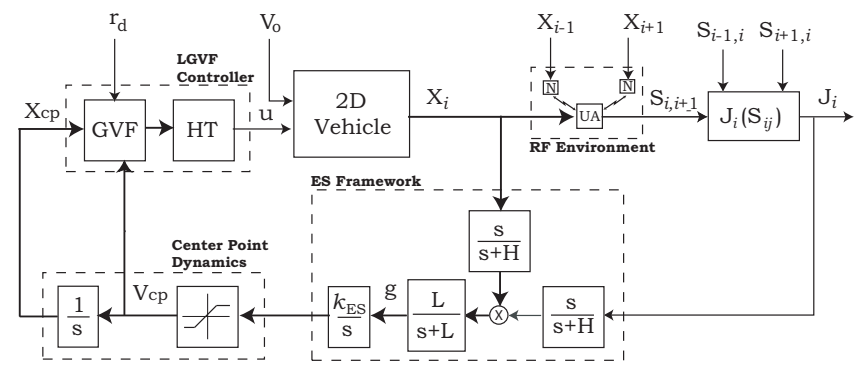

Fig. 2. Decentralized ES algorithm for a $2 D$ kinematic vehicle using a LGVF controller to provide the orbital motion of the vehicle about a virtual center point driven by the gradient estimate of the performance of a communication chain.

The most significant difference in the design of this ES algorithm is that it is a self-exciting system. That is, there is a natural limit cycle that persists in the system (the orbital motion of the vehicle) and this limit cycle provides the required dither signal into a measureable performance function. Because the limit cycle that exsists due to the plant dynamics generates the sinusoidal dither signal, the performance and stability of the controller are dependent upon the performance capabilities of the vehicle. Thus to maintain stability of the ES chaining algorithm, appropriate values for the ES filters, the ES feedback gain $k_{E S}$, and the maximum center point velocity

\footnotetext{
${ }^{1} D_{\theta}^{i}(\cdot)$ denotes the $i^{t h}$ directional derivative of $J$ w.r.t. $\theta$.
}

must be designed for each different vehicle type with different performance abilities.

\section{A. Kinematic Vehicle Model}

It is assumed that the robotic nodes in the network are equipped with a low-level control system that presents a 2-D kinematic model for use by the higher-level ES algorithm. Let $\mathbf{p}_{j} \in \mathbb{R}^{2}$, denoted as $\mathbf{p}_{j}=\left[x_{j}, y_{j}\right]^{T}$, be the position of vehicle $j$ with inertial speed $\left[\dot{x}_{j}, \dot{y}_{j}\right]^{T} \in \mathbb{R}^{2}$ that evolves according to the standard (Cartesian) bicycle-like kinematic model

$$
\begin{aligned}
\dot{x}_{j} & =v_{j} \cos \psi_{j} \\
\dot{y}_{j} & =v_{j} \sin \psi_{j} \\
\dot{\psi}_{j} & =v_{j} c_{j}
\end{aligned}
$$

where $\left[x_{j}, y_{j}\right]^{T} \in \mathbb{R}^{2}$ is the two-dimensional inertial position of node $j, \psi_{j} \in[0,2 \pi)$ is the track angle (compass heading), $v_{j}$ is the commanded speed (held constant), and $c_{j}$ is the bounded path curvature. The bicycle kinematic model is chosen over a unicycle model because this model covers a wider class of 2D nonholonomic vehicles, moving in only a forward direction and that cannot turn on the spot, such as bicycles, cars, and autonomous underwater and aerial vehicles [11].

It should be noted that the major difference of the bicyclelike model from the unicycle model is that the heading rate is a function of the vehicle speed and the curvature constraints of the vehicle. For bicycles, the curvature is related directly to the steering angle of the front wheel. For an aircraft in a steady-state coordinated turn, the path curvature is

$$
c(v)=\frac{g \tan \phi}{v^{2}},
$$

where $\phi$ is the aircraft bank angle and $g$ is the gravitational constant.

Due to vehicle performance constraints, the path curvature for a vehicle is bounded by upper and lower limits. For an aircraft at a speed $v$,

$$
\omega_{\max }(v)=\frac{g \tan \phi_{\max }}{v}
$$

where $\phi_{\max }$ is the maximum bank angle of the vehicle at speed $v$. Thus the steering input into vehicle $j$ is bounded such that $\left|u_{j}\right| \leq \omega_{\max }$ and gives a minimum orbital radius of

$$
r_{\min }(v)=\frac{v}{\omega_{\max }(v)} .
$$

For bicycles and car-like vehicles, the path curvature bound is directly related to the physical limitations in the motion of the steering wheels.

This minimum radius, as will be seen later, is the effectively the lower bound on the final error (or distance) of the vehicle from the optimal communication location, which will be the location of orbit center point for the loiter circle. While the orbit center point can be driven to the location of optimal communication, the robitc relay will always be at best no closer than $r_{\text {min }}$. 
Because of the wide range of dynamics and physical constraints of different types of robotic nodes, it is not practical to drive the vehicle speed or heading directly by the ES dither signal as done in [14]. Instead a Lyapunov guidance vector field (LGVF) controller is used to drive the vehicle to an orbital (limit cycle) motion about a center point. The center point is then driven with vitural point mass dynamics by the ES framework in the chaining algorithm.

\section{B. Lyapunov Guidance Vector Field Controller}

To provide the sinusoidal perterbation signal required by the ES framework, A Lyapunov guidance vector field (LGVF) controller [13] is used to drive the vehicle to a circular limit cycle about a virtual center point, $\mathbf{p}_{c p} \in \mathbb{R}^{2}$. Since the vehicle is orbiting $\mathbf{p}_{c p}$, the ES framework does not drive the vehicle directly to the optimal communication location, but instead pushes $\mathbf{p}_{c p}$ to the optimal communication location that the vehicle orbits about using the LGVF controller.

The LGVF controller is split into two components, a guidance vector field (GVF) generator and a heading tracker (HT) controller. The heading tracker drives the robotic relay to the desired loiter circle at a radial distance of $r_{d}$ from the orbit center point $\mathbf{p}_{c p}=\left[x_{c p}, y_{c p}\right]^{T}$ as given by the generated vector field

$$
\begin{aligned}
\mathbf{f}\left(\mathbf{p}_{r}\right)= & {\left[\begin{array}{l}
\dot{x}_{d} \\
\dot{y}_{d}
\end{array}\right] } \\
= & \beta\left[\begin{array}{cc}
-\left(r^{2}-r_{d}^{2}\right) & -2 r r_{d} \\
-2 r r_{d} & -\left(r^{2}-r_{d}^{2}\right)
\end{array}\right]\left[\begin{array}{l}
x-x_{c p} \\
y-y_{c p}
\end{array}\right] \\
& +\left[\begin{array}{c}
\dot{x}_{c p} \\
\dot{y}_{c p}
\end{array}\right]
\end{aligned}
$$

where $r^{2}=\mathbf{p}_{r} \cdot \mathbf{p}_{r}=\left(x-x_{c p}\right)^{2}+\left(y-y_{c p}\right)^{2}$ is the squared radial distance of the UAV from the loiter center point, $\mathbf{p}_{c p}$, $\beta$ is a non-negative scalar that guarantees convergence to the desired loiter circle when the center point is moving [13], and $\mathbf{v}_{c p}=\left[\dot{x}_{c p}, \dot{y}_{c p}\right]^{T}$ is the center point velocity.

The guidance vector field gives the desired velocity, which is used to generate a turn rate command to the vehicle through the HT. Let $e_{\psi}=\psi-\psi_{d}$ where $\psi_{d}$ is the desired compass heading given as

$$
\psi_{d}=\arctan \left(\frac{\dot{y}_{d}}{\dot{x}_{d}}\right) .
$$

The heading angle error is driven to zero by the turn rate command

$$
\omega=\dot{\psi}_{d}-\lambda \cdot\left(\psi-\psi_{d}\right)
$$

where

$$
\dot{\psi}_{d}=\frac{v}{r_{d}} .
$$

This controller is globally stable limit cycle about $\mathbf{p}_{c p}$ and is stable for any value of $\mathbf{v}_{c p}$. However, since it is assumed that the vehicles have bounded turn rate capabilities, $r_{d}$ should be chosen such that $r_{d}<r_{\min }$.

\section{Center Point Dynamics}

For the ES framework to be stable, and to generate the appropriate gradient estimate, the system needs to exhibit three different time scales [21] :

1) Fast - tracking of the center point

2) Medium - the orbital motion

3) Slow - the LPF filter in the ES

Since the amplitude and excitation frequency of the perturbation signal are set by $v$ and $r_{d}$, the fast and slow dynamics must also be functions of the vehicle performance. Due to the speed constraints placed on the dynamics of the center point, the convergence rate of the center point to the optimal location is bounded by the maximum speed of the center point.

For the error dynamics of the center point to be fast, and to maintain the cyclic orbit about the center point, the motion of the center point must be slow as compared to the speed of the vehicle, i.e. $v_{c p}<<v_{j}$. In the ES chaining algorithm the center point velocity is bounded by $V_{c p}$ so that $v_{c p} \leq V_{c p}$ and $V_{c p}<<v_{j}$.

It should be pointed out that center point velocity saturation is required in the loop because even though we can choose $k$ small enough that the speed of the orbit center point remains slow, as compared to the UA for a given environment and performance function, the output of the ES framework depends upon the magnitude and shape of the performance function, which is not necessarily known a priori. Thus, there could be unexpected environments in which if the center point speed was not bounded, it could reach the maximum flight speed of the aircraft. At this point, the motion of the UA about the center point is no longer cyclic and is not generating the periodic perturbation signal of the performance function required for the ES framework to generate the gradient estimate.

\section{Electronic Chaining Simulation Results}

In this section, simulations of the decentralized ES electronic chaining algorithm for the control of a team of unmanned aircraft are presented. Simulations of electronic chaining using the gradient directly are not presented in this paper since in physical environments the true gradient will not be known, so it is of interest to present results of the ES controller which can be used in any unknown environment. For the simulations, the aircraft are limited to a maximum 30 degree bank angle, flying at $25 \mathrm{~m} / \mathrm{s}$, and their ordering is preset and maintained depending upon the starting location of the UAs. The maximum center point velocity is set to $5 \mathrm{~m} / \mathrm{s}$.

Though it is not know by the ES controller, the radios in the simulation are assumed to follow the standard exponential decay model

$$
P_{i j}=P_{i j}=K_{r} d_{i j}^{-\alpha}
$$

where $K_{r}$ is the link gain, $d_{i j}$ is the separation distance of the receiver from the transmitter, $\alpha$ is the exponential decay rate, and $P_{i j}$ is the received power. The radio values are set to $K_{r}=3822$ and $\alpha=3.5$. For the simulation with a noise source, the noise source is taken to be a faulty radio transmitting with $K_{r}=382$. Note, the choice of using the exponential 

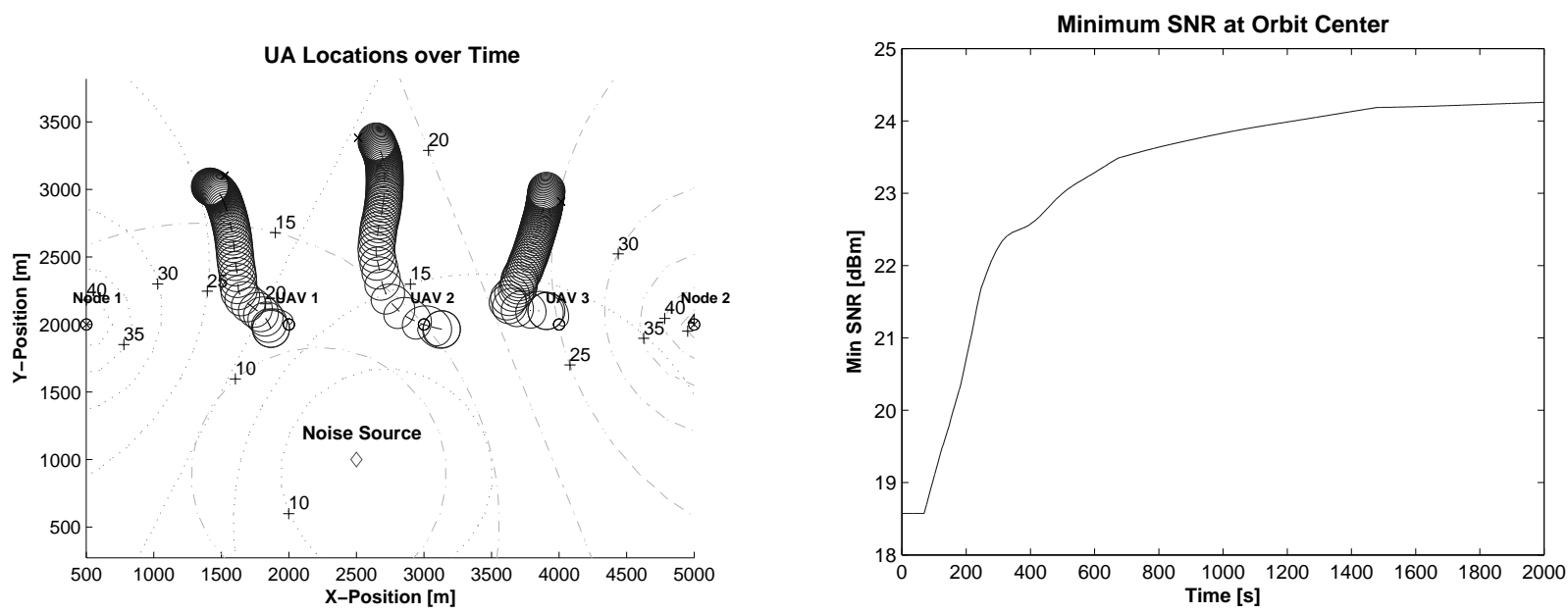

Fig. 3. Simulation of three (3) UA relay nodes reacting to a localized noise source. (a) Motion of UAs within the environment also showing noise source location and the SNR contours of the two end nodes. (b) The minimum SNR value along the chain during the simulation.
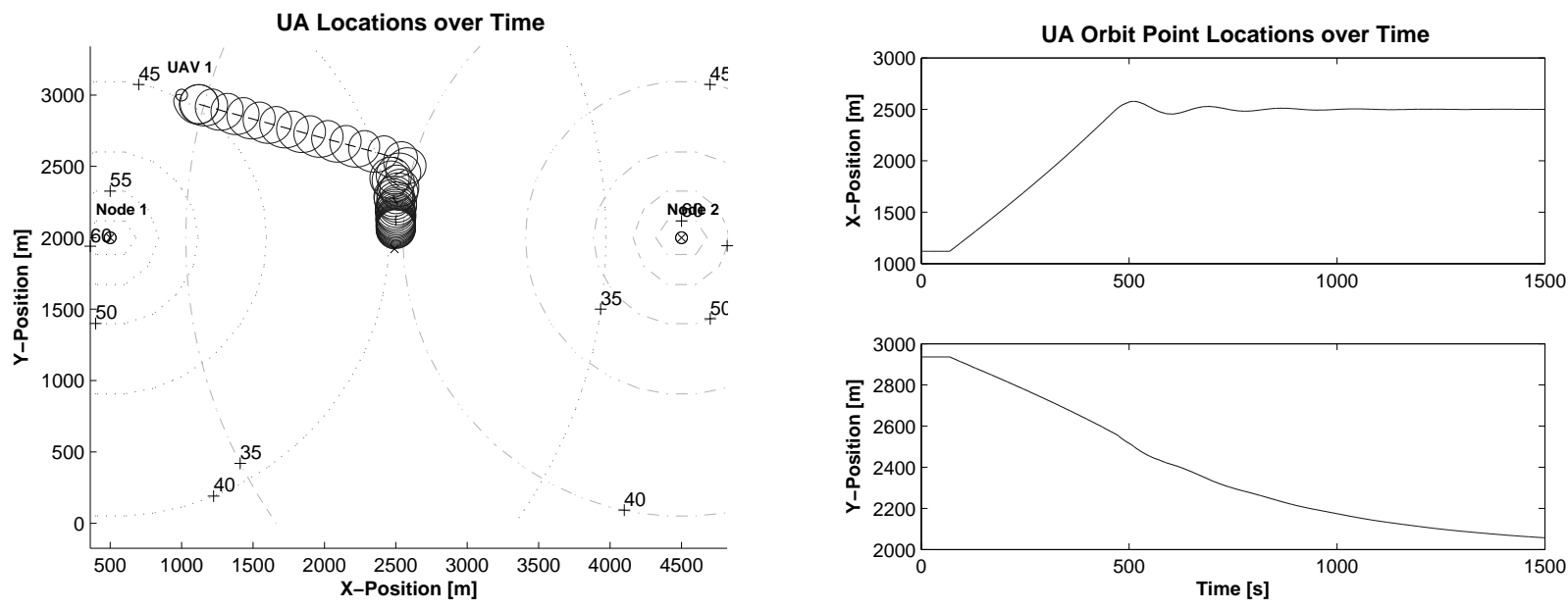

Fig. 4. Location of the orbit center point for a single UA and no localized noise showing the linear (for $t \in(50,500) \mathrm{s}$ ) and asympototic convergence (for $t>500 \mathrm{~s}$ ) of the UA location to the optimal X-Y location

decay model was for ease of programming in the simulation. However, by assuming that the noise source is a faulty radio that is acting as a jamming node, even in the simulation the SNR models are no longer concetric circles about the radio nodes. Instead, depending upon the power and location of the a noise source the SNR contour lines are extremely skewed and non-symmetric.

Figure 3 shows a simulation run with three UAs and two static end nodes, with Fig. 3a being a top down view of the simulation environment and Fig. $3 b$ is the minimum link SNR along the chain. At the beginning of the simulation, the UA relays are aligned along the chain as would be a result of running a position based controller such as in [2]. At time $t=0 \mathrm{~s}$ a noise source located at $[2500,1000] \mathrm{m}$ is introduced. Since position based controllers would not sense the change in the RF environment, the nodes would maintain their current position. However, using the electronic chaining algorithm the figure shows that the UAs react appropriately to the jamming signal source and form a bowed communication chain. Figure $3 \mathrm{~b}$ shows that at $t=0 \mathrm{~s}$ the minimum SNR at the orbit center points along the communication chain was less then $19 \mathrm{dBm}$ and that the electronic chaining controller was able to improve the minimum value to above $24 \mathrm{dBm}$ by moving the location of the vehicles orbit center point.

Figure 4 shows results from a simulation with a single UA, two end nodes and no localized no source. In Fig. $4 \mathrm{a}$, the position of the UA and the center point are shown. From this figure one can see that when the UA was far away, it headed directly in the direction of improving the minimum SNR (which is the SNR from the far right node) at the maximum speed of the center point. Figure $4 \mathrm{~b}$ shows just the $\mathrm{X}-\mathrm{Y}$ position of the orbit center point is shown to highlight the bounded convergence rate of the ES algorithm. For $t \in(50,500) \mathrm{s}$ the positional errors (especially on the Y- 
axis) show the bounded convergence rate due to the bounded center point speed.

\section{CONCLUSION}

In this paper a definition of an optimal communication chain of relay nodes in an ad hoc network was presented based on the SNR instead of relative position. By using the SNR instead of position, a communication chain of robotic relays can respond to changes and unexpected features in the RF environment that is not possible with position based chaining solutions.

Since the operating environment is generally not known a priori to deployment of a network, an adaptive model-free ES chaining algorithm was presented to control the motion of $2 \mathrm{D}$ nonholonomic vehicles acting as communication relays. Even without specific knowledge of the SNR field, the ES algorithm is able to drive the team of vehicles to an optimal locations with only local measures of the SNR. The mobility of the vehicle was modeled as a bicycle-like kinematic model and is chosen over the unicycle model because the model covers a wider class of 2D nonholonomic vehicles, including unmanned aircraft. An orbital motion of the vehicle due to a LGVF controller was applied to extremum seeking in a unique way in that the orbital motion of the vehicle about an orbit center point generated the dither and demodulation signals required by the ES algorithm. A specific application using UAs was and simulated to highlight the fact that the performance of the ES algorithm is limited due to the performance constraints and capabilities of the individual vehicles within the chain.

Future work will include varying the aircraft flight speed so as to change the orbiting radius to improve the performance of the ES framework. By slowing the aircraft down when it approaches the optimal location, a smaller orbital radius can be tracked and the aircraft will generate a smaller dither signal and will improve the estimation of the optimal communication location. In addition, since the convergence rate is bounded by the flight speed of the aircraft, it is desirable to have the UA fly close to its maximum flight speed when the center point is far away from the optimal location.

\section{REFERENCES}

[1] C. Dixon and E. W. Frew, "Maintaining a linked network chain utilizing decentralized mobility control," in AIAA's Guidance, Navigation and Control Conference and Exhibit. AIAA, 21 - 24 Aug 20062006.

[2] D. Goldenberg, J. Lin, A. S. Morse, B. E. Rosen, and Y. R. yang, "Towards mobility as a network control primitive," in Mobihoc '04. ACM, 24-26 May 2004.
[3] C. Dixon, E. Frew, and B. Argrow, "Radio leashing of an unmanned aircraft," in AIAA's Infotech@Aerospace Conference, 26-29 Sep. 2005.

[4] C. Dixon and E. W. Frew, "Controlling the mobility of network nodes using decentralized extremum seeking," in 45th IEEE Conference on Decision and Control. IEEE, 11-12 December 2006.

[5] R. W. Beard and V. Stepanyan, "Synchronization of information in distributed multiple vehicle coordinated control," in IEEE Conference on Decision and Control. IEEE, December 20032003.

[6] R. Olfati-Saber and R. M. Murray, "Flocking with obstacle avoidance: Cooperation with limited information in mobile networks," in Conference on Decision and Control (CDC), 2003.

[7] L. Yang, P. K.M., and P. M, "Stability analysis of m-dimensional asynchronous swarms with a fixed communication topology," IEEE Transactions on Automatic Control, vol. 48, no. 1; analyzed. Such stability analysis is of fundamental importance, pp. 76-95, December 2003.

[8] J. Sweeney, T. Brunette, and Y. Y. R. Grupen, "Coordinated teams of reactive mobile platforms," in International Conference on Robotics and Automation. IEEE, May 11-15 2002.

[9] J. Cortes, S. Martinez, T. Karatas, and F. Bullo, "Coverage control for mobile sensing networks," in IEEE Transactions on Robotics and Automation, May 2002 2004, pp. 1327-1332.

[10] P. Basu and J. Redi, "Movement control algorithms for realization of fault-tolerant ad hoc robot networks," Network, IEEE, vol. 18, no. 4, p. 36, 2004, 0890-8044.

[11] G. Indiveri, "Kinematic time-invariant control of a $2 \mathrm{~d}$ nonholonomic vehicle," in 38th Conference on Decision and Control (CDC'99), December 1999

[12] L. E. Dubins, "On curves of minimal length with a constraint on average curvature, and with prescribed initial and terminal positions and tangents," American Journal of Mathematics, vol. 79, no. 3, pp. 497-516, jul 1957.

[13] E. W. Frew and D. Lawrence, "Cooperative stand-off tracking of moving targets by a team of autonomous aircraft," in AIAA Guidance, Navigation, and Control Conference, August 2005.

[14] C. Zhang, D. Arnold, N. Ghods, A. Siranosian, and M. Krstic, "Source seeking with nonholonomic unicycle without position measurement part i: Tuning of forward velocity," in 45th IEEE Conference on Decision and Control, 13-15 December 2006.

[15] G. Kramer, M. Gastpar, and P. Gupta, "Cooperative strategies and capacity theorems for relay networks," IEEE Transactions on Information Theory, vol. 51, no. 9, pp. 3037-3063, September 2005.

[16] B. Taub and D. L. Schilling, Principles of Communication Systems. New York: McGraw-Hill, 1986.

[17] F. H. Clarke, "Generalized gradients and applications," Transactions of the American Mathematical Society, vol. 205, no. (Apr. 1975), pp. 247262, 1975.

[18] A. Bacciotti and F. Ceragioli, "Stability and stabilization of discontinuous systems and nonsmooth lyapunov functions," ESAIM. Control, Optimization \& Calculus of of Variations, vol. 4, pp. 361-376, 1999.

[19] K. B. Ariyur and M. Krstic, Real-Time Optimization by ExtremumSeeking Control. New York, NY: John Wiley and Sons Pub., 2003.

[20] K. Ariyur and M. Krstic, "Multivariable extremum seeking feedback: Analysis and design," in Fifteenth International Symposium on Mathematical Theory of Networks and Systems, August 12-16 2002.

[21] M. Krsti and H. H.Wang, "Stability of extremum seeking feedback for general nonlinear dynamic systems," Automatica, vol. 36, p. 595601, 2000 . 